\title{
Simulation of Train Load on Deformation of Big- Diameter Shield Tunnel
}

\author{
C.J. Chen \\ China Railway Tunnel Survey \& Design Institute Co., Ltd, \\ Tianjin, 300133
}

\author{
H.L. Zhu \\ Key Laboratory of Road and Traffic Engineering of the \\ Ministry of Education, Tongji University, Shanghai, 201804
}

\begin{abstract}
In order to study the impact of train load on deformation of big-diameter shield tunnel, the Vehicle-TrackTunnel-Soil coupling dynamic model was established, and the reaction force of fasteners was used to transmit between the Vehicle-Track coupling dynamic model and the Tunnel-Soil finite element model. The results are as follows: the changing of vertical cross section deformation and horizontal cross section deformation has congruent relationship with the changing of train load and there exists a time difference; under the impact of train load, tunnel size increases in vertical direction and shrinks in horizontal direction, cross section shape grows into vertical oval and the deformation is confined to a very small amount.
\end{abstract}

Keywords-shield tunnel; big diameter; cross section deformation; coupling dynamic model

\section{INTRODUCTION}

With the fast development of urban railway system, more and more shield tunnels have been put into operation. According to the safety inspection on service shield tunnels, cross section deformation is the most common safety problem for tunnel deterioration is close related to it. Wang and Zhang [1] found leakage will be triggered once the cross section deformation exceeds a limiting value, and the worse is that segments will be crushed, bolts lost function, and the collapse of the whole tunnel; the safety problem will be more seriously in large-diameter shield tunnel. Tunnel deformation happens both in construction and operation period, the former is mainly caused by disturbance of shield construction and the latter is influenced by long-term operation load, leakage and surrounding engineering event. Existing research on shield tunnel deformation can be classified into three aspects: the surrounding engineering events and tunnel leakage on tunnel deformation and internal force $[2,3,4,5]$; the qualitative analysis on tunnel deformation based on field test data[6,7]; the elastic limit radius of curvature based on Modified Routine Method and the relationship between cross section deformation and segment internal force[8]. The impact of operation load on tunnels is mostly given on tunnel vibration: the dynamic response analysis on shield tunnel in operation period using three-dimensional dynamic finite difference method [9]; dynamic response analysis on lining structures of tunnels with three kinds of cross section [10]; the study on the roadbed and standard segments in Shanghai based on monitoring data [11].
Aforementioned research are mainly focus on conventional diameter tunnel and the influence of operation traffic load which is a significant factor on tunnel cross section deformation can be rarely seen. With the VehicleTrack-Tunnel-Soil coupling dynamic method, this paper studies effect of operation load on cross section deformation of large-diameter shield tunnel.

\section{CALCULATION METHOD AND MODEL}

The Vehicle-Track-Tunnel-Soil coupled dynamic model consists of two main parts: Vehicle-Track coupling dynamic model and Tunnel-Soil finite element model. The reaction force of fasteners is used to transmit forces between the two constituent parts. A-Train with six cars is adopted and there are three kinds of operation train load: on the right line, on the left line and on both lines, of which the last one is the most unfavorable and is used in the model.

\section{A. Vehicle-Track Coupling Dynamic Model}

The classical model of vehicle-track vertical coupling dynamics, [12], is used. In the vehicle model, each section of the vehicle is modeled as a multi-rigid-body system with seven rigid bodies and 10 degrees of freedom, considering four wheel sets, two bogies and one car body. The dynamic equation of the vehicle can be expressed as unified format:

$$
\left[M_{v}\right]\{\ddot{X}\}+\left[C_{v}\right]\{\dot{X}\}+\left[K_{v}\right]\{X\}=\left\{Q_{v}(t)\right\}
$$

Where $\{\ddot{X}\},\{\dot{X}\}$ and $\{X\}$ are, respectively, the generalized acceleration vector, the generalized velocity vector and the generalized displacement vector; $\left[M_{v}\right]$, $\left[C_{v}\right]$ and $\left[K_{v}\right]$ are, respectively, the mass matrix, damping matrix and the stiffness matrix; $\left\{Q_{v}(t)\right\}$ is the generalized load vector in the vehicle model.

In the model, the rail is modeled as an infinite Euler beam lying on the elastic discrete points of support. The elasticity mainly depends on the plate under the rail for the doubleblock non-ballast track. The equation of vibration mode coordinate for rail is given by:

$$
\left[M_{r}\right]\{\ddot{q}\}+\left[C_{r}\right]\{\dot{q}\}+\left[K_{r}\right]\{q\}=\left\{P_{r}\right\}
$$

where $\left[M_{r}\right],\left[C_{r}\right]$ and $\left[K_{r}\right]$ are respectively the generalized mass matrix, the generalized damping matrix and the generalized stiffness matrix; $\{\ddot{q}\},\{\dot{q}\}$ and $\{q\}$ are 
respectively the generalized acceleration vector, the generalized velocity vector and the generalized displacement vector; $\left\{P_{r}\right\}$ is the generalized load vector of the rail mode of vibration coordinate of the rail vibration mode coordinates in the system. The acceleration of the rail is expressed as:

$$
\left\{\ddot{X}_{r}(x)\right\}=[Y(x)]\{\ddot{q}\}
$$

Where $\left\{\ddot{X}_{r}(x)\right\}$ is the acceleration vector of rail and $[Y(x)]$ is the rail mode matrix of vibration.

Hertz nonlinear elastic contact theory is adopted to solve the wheel-rail contacting relationship, and the wheel-rail vertical force is expressed as:

$$
p(t)=\left\{\left[Z_{w j}(t)-Z_{r}\left(x_{w j}, t\right)-Z_{0}(t)\right] / G\right\}^{3 / 2}
$$

Where $\mathrm{G}$ is the contacting constant; $Z_{w j}(t)$ represent the displacement of the $\mathrm{j}$ wheel in moment of $\mathrm{t}, Z_{r}\left(x_{w j}, t\right)$ is the rail displacement at the location of the $\mathrm{j}$ wheel in moment of $\mathrm{t}$, $Z_{0}(t)$ is the random irregularity of rail.

The force of fasteners in the vehicle-track coupling dynamic model is expressed as:

$$
\left\{F_{r s}\right\}=\left[C_{p}\right]\left\{\dot{X}_{r}\left(x_{i}\right)\right\}+\left[K_{p}\right]\left\{X_{r}\left(x_{i}\right)\right\}-\left[C_{p}\right]\left\{\dot{X}_{F E M}\right\}-\left[K_{p}\right]\left\{X_{F E M}\right\}(5)
$$

where $\left\{F_{r s}\right\}$ is the force vector of fasteners; $\left[C_{p}\right]$ and $\left[K_{p}\right]$ are respectively the damping matrix and the stiffness matrix of the plate under the rail; $\left\{\dot{X}_{r}\left(x_{i}\right)\right\}$ and $\left\{X_{r}\left(x_{i}\right)\right\}$ are respectively the rail velocity vector and the displacement vector at the fastener points, $\left\{\dot{X}_{F E M}\right\}$ and $\left\{X_{F E M}\right\}$ are respectively the velocity vector and the displacement vector of the deck slab at corresponding fastener points in the FEM. The force of fasteners in the model is shown in figure 1 .

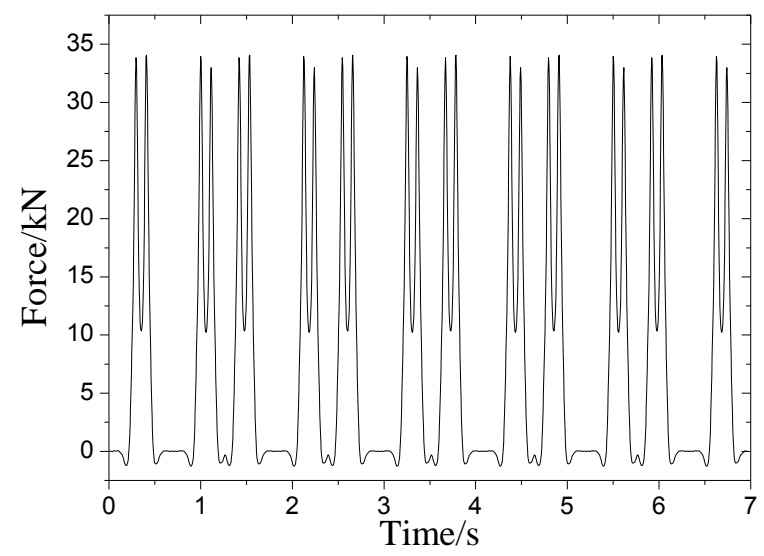

FIGURE I. TIME HISTORY OF FASTENERS FORCE

\section{B. Tunnel-Soil FEM}

The external diameter and internal diameter of the tunnel are respectively $11.2 \mathrm{~m}$ and $10.2 \mathrm{~m}$ and the soil layer which the tunnel passing through is mainly silty-fine sand; width of the segment is $2 \mathrm{~m}$, and the thickness of the segment is $0.5 \mathrm{~m}$. One segment ring contains one top block, two adjacent blocks and five standard blocks; segment rings are jointed together by 33 T30 bolts in circumferential direction and 16 T36 bolts in longitudinal direction. Three dimensional solid bolt elements are used and bolt force is given to simulate bolt [18]. Material properties are listed in table 1.

TABLE I. MATERIAL PARAMETERS FOR MODEL.

\begin{tabular}{|c|c|c|c|}
\hline Material & Es/ $\mathbf{E}_{\mathbf{0}}(\mathbf{M P a})$ & Density $\left(\mathbf{k g} / \mathbf{m}^{\mathbf{3}}\right)$ & Poisson's ratio \\
\hline Soil & 14.5 & 1900 & 0.3 \\
\hline Segment & 34500 & 2500 & 0.2 \\
\hline Roadbed & 30000 & 2400 & 0.2 \\
\hline Bolt & 200000 & 7850 & 0.167 \\
\hline
\end{tabular}

Inhomogeneous model which has the overall-detailoverall characteristic in longitudinal direction is adopted in the paper in order to achieve a good computational accuracy and high computational efficiency. For the middle three segment rings, as is shown in Figure 2, segment joints are taken into consideration; and the two end parts of the segment is simplified as an overall structure by reducing stiffness of segment referring to Modified Routine Method so to simulate the reducing of segment ring stiffness. Mesh of segment is shown in Figure 3.

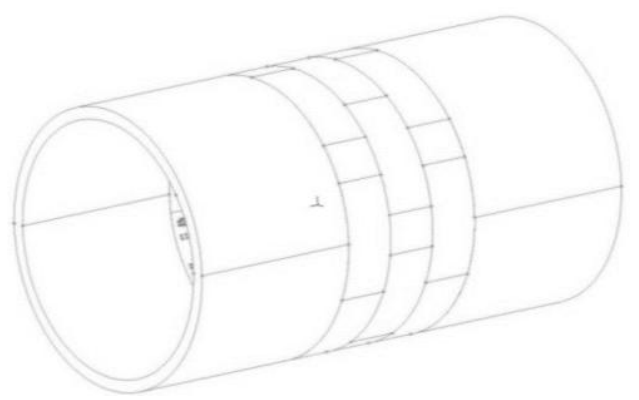

FIGURE II. TUNNEL MODEL.

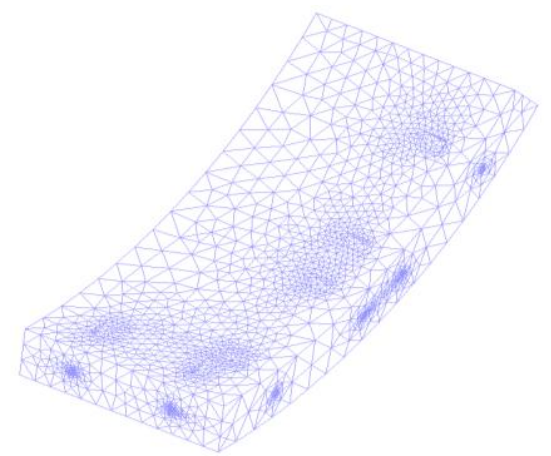

FIGURE III. SEGMENT MESH.

The surface-surface contact element is used in the FEM which contains several kinds of contacts: segment-segment contact, segment-bolt contact, segment-roadbed contact, segment-soil contact. The tangential friction coefficient of segment-segment contact set at $0.83[13,14]$, to consider the frictional resistance of rubber pad at segment joints, and that of segment-bolt contact is set as $0.3[15]$ and segment-soil contact is $0.4[16]$. The fixed contact of segment- roadbed contact and segment-bolt end contact is realized by Tied Contact [17] which considers the displacement continuity between two contacting surfaces. 


\section{CALCULATION RESULTS ANALYSIS}

\section{A. Cross Section Deformation}

The vertical cross section deformation is defined as the relative displacement changing between two points at vault and arch bottom, and it is shown in Figure 4. The horizontal cross section deformation is defined as the relative displacement changing between two opposite points at haunch, and it is shown in Figure 5.

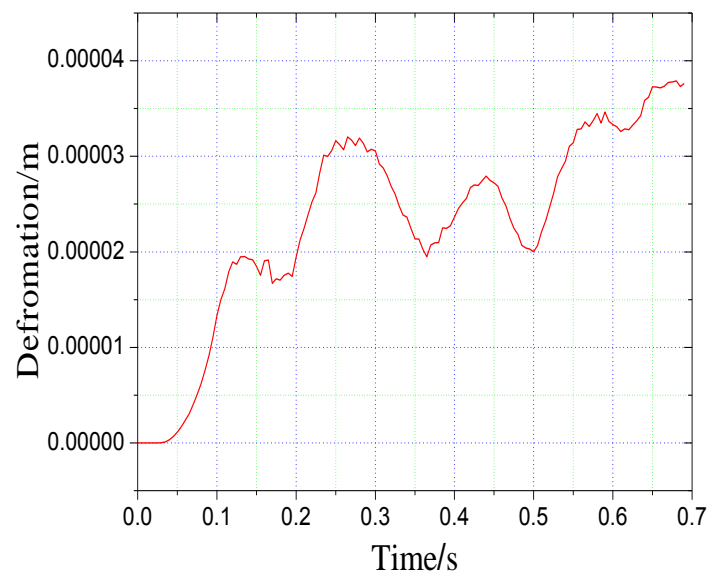

FIGURE IV. VERTICAL DEFORMATION.

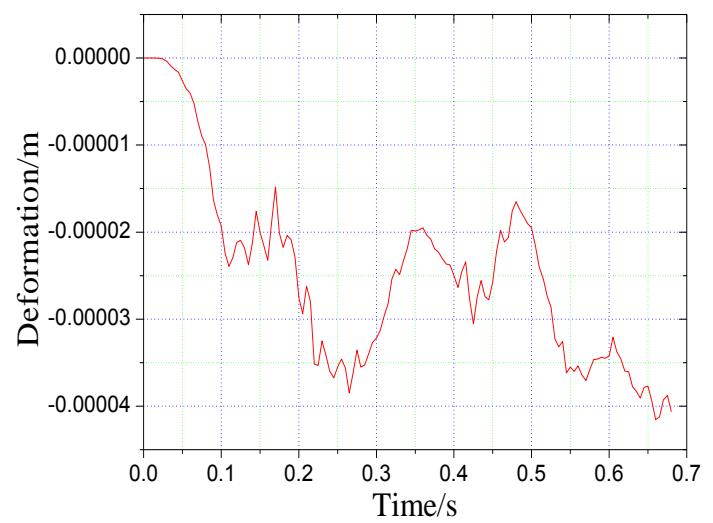

FIGURE V. HORIZONTAL DEFORMATION.

It can be seen in Figure 4 the vertical deformation curve is fluctuant and contains three periods: 1st rising stage, equilibrium stage and 2 nd rising stage. In the whole period there exists correspondence with little phase difference between the vertical deformation and load spectrum (Figure 1): the 1st and 2 nd rising stage can be seen as the responses of 1 st and 2 nd bogie and the two crests in each rising stage are corresponding to the two wheel sets in each bogie. More can be gained from Figure 4 that as the bogie approaching the surveying point, the deformation increases to two crests in 1st rising stage; as the bogie leaving away from the surveying point, the deformation keeps balance with fluctuation; then it comes to 2nd rising stage. In the equilibrium stage, the phenomenon that the vertical deformation first decreases then increases and decreases at last can be explained that two bogies move in the vicinity of the surveying point.
From Figure 5, the law is similar with vertical deformation that there exists 1st declining stage, equilibrium stage and 2nd declining stage; and there are two troughs in each declining stage reciprocally corresponding two the wheel sets in each bogie.

It can be summarized from Figure 4 and Figure 5 that vertical deformation and horizontal deformation reciprocally corresponds to each other; the cross section changes into a vertical ellipse influenced by operation load; the cross section deformation accumulates as time grows.

\section{B. Tunnel Displacement}

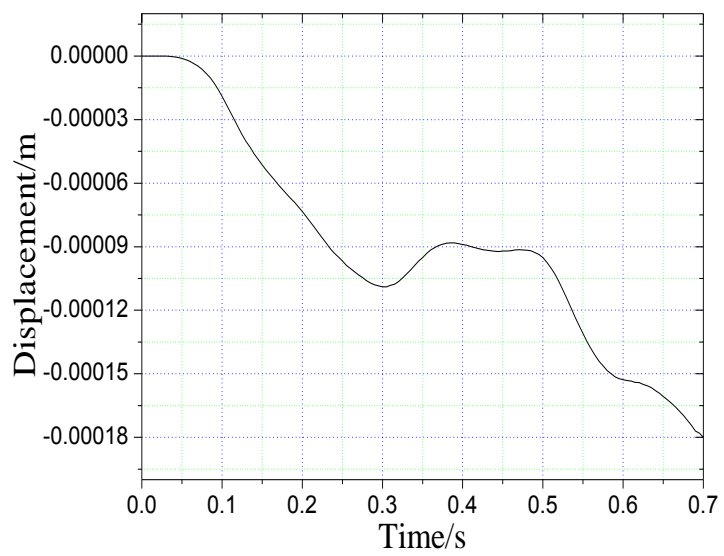

FIGURE VI. TUNNEL DISPLACEMENT.

Figure 6 shows the displacement of tunnel caused by train load, and the negative value means settlement. It can be seen tunnel settlement reaches the first valley value as the first bogie passing; then settlement decreases little and keeps at a stable level; when the second bogie passes, the settlement increases again and reaches the maximum value. The phenomenon that settlement decreases little and keeps stable can be explained as following: after the first bogie leaves the surveying point and before the second bogie arrives, the total load increases and has a whole effect on the surveying point. Elastic material is used in the model, so the accumulation of settlement is a result of the increasing of load.

\section{CONCLUSION}

Coupling dynamic model was used to study the effect of train load on tunnel deformation and the conclusions are as follows:

In the whole process, both the vertical deformation and horizontal deformation experience three periods: 1st rising stage, equilibrium stage and 2 nd rising stage;

Vertical deformation and horizontal deformation corresponds to each other and both match up with load spectrum; the cross section tends to be vertical oval under train load.

Under train load which is a cyclic loading, tunnel settlement and cross section deformation will accumulate with time and further work should be done to get a better understanding of this sophisticate problem. 


\section{REFERENCES}

[1] Wang Ru-lu, Zhang Dong-mei. Mechanism of transverse deformation and assessment index for shield tunnels in soft clay under surface surcharge. Chinese Journal of Geotechnical Engineering, 35(6), pp.1092-1101, 2013.

[2] WANG Yang, HE Chuan, ZENG Dong-yang, etc. Model Test and Numerical Simulation of Influence of Perpendicular Undercross Shield Tunnel Construction on Existing Tunnel. JOURNAL OF THE CHINA RAILWAY SOCIETY, 32(2), pp. 79-85, 2010.

[3] Liu Tingjin. Study on Shield Tunnel Deformation Due to Foundation Pit Construction. CHINESE JOURNAL OF ROCK MECHANICS AND ENGINEERING, 27(z2), pp. 3393-3400, 2008.

[4] Wei Gang. Measurement and analysis of impact of foundation pit excavation on below existed shield tunnels. Rock and Soil Mechanics, 34(5), pp. 1421-1428, 2013.

[5] Liu Yin, Zhang Dongmei, Huang Hongwei. Influence of long-term partial drainage of shield tunnel on tunnel deformation and surface settlement. Rock and Soil Mechanics, 34(1), pp. 290-298, 2013.

[6] Huang Xiaoping, Yang Xin'an, Lei Zhenyu, et al. On Convergent Deformation of the Existing Metro Tunnel. URBAN MASS TRANSIT, 12(3), pp. 55-58, 2009.

[7] Ye Yaodong, Zhu Hehua, Wang Rulu. Analysis on the Current Status of Metro Operating Tunnel Damage in Soft Ground and Its Causes. CHINESE JOURNAL OF UNDERGROUND SPACE AND ENGINEERING, 3(1), pp. 157-160, 2007.

[8] Wang Zhiliang, Shen Linfang, Liu Guobin. Research on Joints Properties for Tunnel Convergence Deformation of Operating Metro. Journal of Kunming University of Science and Technology(Science and Technology), 38(2), pp. 38-43, 2013.

[9] Mo Haiou, Deng Feihuang, Wang Junhui, et al. Analysis of Dynamic Responses of Shield Tunnel During Metro Operation. Chinese Journal of Rock Mechanics and Engineering, 25(z2), pp. 3507-3512, 2006.

[10] Wang Xiangqiu, Yang Linde, Zhou Zhiguo. Dynamic Response Analysis of Lining Structure for Tunnel under Vibration Loads of Train. Chinese Journal of Rock Mechanics and Engineering, 25(7), pp.1337-1342, 2006.

[11] Li Mingyu, Liu Guobin, Hu Mengda, et al. Field Measurement and Analysis of Structural Vibration Response in the Metro Shield Tunnel. JOURNAL OF THE CHINA RAILWAY SOCIETY, 33(6), pp. 88-93, 2011.

[12] Zhai Wanming. Vehicle-Track coupling dynamics (Third Edition). Science Press, Beijing, 2007.

[13] Li, Yujie, He Ping, Qin Dongping. Stress analysis of metro shield tunnel segment. CHINA CIVIL ENGINEERING JOURNAL, 44, pp. 131-134, 2011.

[14] He Taihong, Wang Binglong, Liu Jianguo, et al. Rational Determination of Elastic Gasket Contact Stress and losure Pressure for Shield Tunnel Segments. URBAN MASS TRANSIT, 12(12), pp. 62-63, 68, 2009.

[15] Chen Junsheng, Mo Haiou. Three-dimensional FEM Analysis on Flexural Rigidity of Segment Joints in Shield Tunnel. JOURNAL OF THE CHINA RAILWAY SOCIETY, 31(4), pp. 87-91, 2009.

[16] BGI ENGINEERING CONSULTANTS LTD. Code for municipal engineering investigation and surveying, CHINA PLANNING PRESS, Beijing, 1994.

[17] ADINA R \& D Inc. Theory and Modeling Guide Volume I: ADINA Solids \& Structures. Watertown: ADINA R \& D Inc, 2005. 